\title{
Employabilité, insertion professionnelle et mobilité des jeunes diplômés dans les métiers de la communication en France
}

Touati Zeineb maître de conférences, Université du Havre CIRTAI touatibz@univ-lehavre.fr Badulescu Cristina, maître de conférences, Université de Poitiers badulescu_cristina@yahoo.com 


\section{Résumé}

De nombreuses études montrent un écart en terme d'accès à l'emploi entre les jeunes diplômés des Grandes Écoles et ceux issus d'un cursus universitaire classique en France. Nous nous intéressons dans cette contribution, selon une analyse comparative, à l'accès à l'emploi ainsi qu'à la corrélation entre le niveau de compétences, le niveau de diplôme et le type d'emploi occupé par les jeunes issus de l'enseignement professionnalisant (Grandes écoles/formations universitaires type DUT ou licence professionnelle) exerçant dans les métiers de la communication.

Mots clés : insertion professionnelle, jeunes, métiers, compétences

\section{Abstract}

Many works in the literature have shown the employment gap between young people with a "Grande Ecole" diploma and University classic diplomas in France. In this paper, we are interested, using a comparative analysis, in the diploma level and employment relationship for young people working in communication with professional studies background (“Grande Ecole”, Universities' diplomas as DUT...).

Keywords: professional insertion, young, job, competencies 
Dans une première partie, nous présenterons brièvement le système d'enseignement supérieur français et les principales formations préparant actuellement aux métiers de la communication. Nous évoquerons également les procédures de sélection des étudiants. Ceci afin de procéder dans une deuxième partie à une étude comparative de l'insertion professionnelle et des poursuites d'études et des orientations des diplômés des Grandes écoles et de celles des diplômés des formations universitaires professionnalisantes et d'observer les stratégies d'amélioration mises en place par les étudiants et les équipes pédagogiques.

\section{L'enseignement et les métiers de la communication en France}

Le système d'éducation en France est centralisé et géré pour les niveaux primaire et secondaire par le ministère de l'éducation nationale et par le ministère de l'enseignement supérieur et de la recherche scientifique pour le niveau supérieur. Les effectifs des élèves et des étudiants avoisinent les 15 millions. Selon les chiffres communiqués en 2010, par le ministère de l'enseignement supérieur, la France formerait 150000 titulaires de master par an.

\subsection{Les formations universitaires et les grandes écoles}

L'offre de formation post baccalauréat en France se décompose en plusieurs types. Une fois son baccalauréat obtenu l'étudiant a le choix entre des formations longues au sein des universités qui proposent des formations dans tous les domaines permettant l'obtention de 3 niveaux de diplômes: la licence $(\mathrm{Bac}+3)$, le master $(\mathrm{Bac}+5)$ et le doctorat $(\mathrm{Bac}+8)$, comme elles permettent l'obtention de diplômes spécialisés notamment dans le domaine de la santé. Ce système a été mis en place récemment dans le cadre de la réforme $\mathrm{LMD}^{59}$. Il y a également des formations courtes type Bac+2 comme le Brevet de Technicien Supérieur (BTS) ou le Diplôme Universitaire de Technologie (DUT) au sein des IUT (Institut Universitaire de Technologie). Les quatre premiers IUT expérimentaux ont été créés en 1965 pour répondre à l'augmentation importante du nombre de bacheliers et pour assurer la diversification de l'offre d'enseignement supérieur. Les IUT étaient chargés, par le décret du 7 janvier 1966, de former en deux années les techniciens supérieurs qui intégraient jusque-là les classes supérieures des lycées techniques. Suite à ce décret sont créés 11 autres IUT accueillant 1503 étudiants. On compte 115 IUT accueillant

\footnotetext{
${ }^{59}$ LMD : Réforme restructurant l'enseignement supérieur en France en 3 en trois niveaux : la Li-
} cence, le Master et le Doctorat. Cette réforme a été mise en place en 2004. 
134000 étudiants en France qui ont depuis leur création formé près d'un million d'étudiants et plus de 3000 diplômés de Licence professionnelle. Les IUT offrent des formations dans les secteurs secondaires comme le génie civil, le génie électrique, le génie mécanique, ou tertiaires comme la gestion des entreprises et des administrations, les carrières sociales et l'information-communication. En plus du DUT, plusieurs IUT offrent des licences professionnelles spécialisées. Les IUT initialement conçus pour accueillir les titulaires de baccalauréat technologique sont devenus des formations sélectives qui attirent de plus en plus les bacheliers titulaires d'une mention (leur proportion passe de 35\% en 1997 à $47 \%$ en 2006). Les effectifs limités et la sélectivité de ces formations rassurent et répondent au besoin d'encadrement souvent exprimé par les bacheliers.

A côté de ces formations, on trouve un ensemble de grandes écoles spécialisées dans l'ingénierie, le commerce et le management, etc. Ces écoles publiques et privées sont majoritairement réputées pour dispenser un enseignement de qualité et pour être des formations exigeantes qui préparent à un diplôme $\mathrm{Bac}+5$. Leur réputation est, entre autres, fondée sur la sélection qui en régule l'accès sur concours et entretien directement après le baccalauréat ou après deux années de classes préparatoires qui sont elles-mêmes des formations sélectives. Plusieurs de ces grandes écoles sont réunies dans des groupements réputés comme les Écoles centrales, les Écoles des mines, les Polytechniques, etc. Le ministère de l'éducation nationale définit une grande école comme étant « un établissement d'enseignement supérieur qui recrute ses élèves par concours et assure des formations de haut niveau ${ }^{60}$ sous la tutelle d'un ministère. Contrairement aux classes préparatoires aux grandes écoles (CPGE) qui dépendent majoritairement du ministère de l'éducation nationale, une grande part des grandes écoles publiques dépendent d'autres ministères (Agriculture, Culture, Défense, Équipement, Industrie, Justice, Santé, etc.).

Les premières grandes écoles ont été créées par l'État au milieu du XVIII ${ }^{\mathrm{e}}$ siècle afin de former des cadres militaires et techniques dans plusieurs secteurs : l'armée, l'administration centrale, les ponts et chaussées, de l'agriculture, etc. Les huit premières écoles supérieures réorganisées dont l'accès est basé sur un concours public, font partie des grands groupes des écoles connues aujourd'hui comme: L'école Polytechnique, l'école des ponts et chaussées, les écoles des Mines, SaintCyr, l'école Navale, les écoles normales supérieures (ENS). Tout au long du XIXème, cette liste a été complétée par des écoles privées comme l'École centrale des arts et manufactures.

En 1819, on note la création de l'École supérieure de commerce de Paris, la première école de commerce en France. Elle sera suivie par deux vagues importantes

\footnotetext{
${ }^{60}$ Arrêté du 27 août 1992 relatif à la terminologie de l'éducation.
} 
de création de plusieurs autres grandes écoles de commerce. La première vague dans les années 1860 et 1870 et la deuxième dans les années 1900. C'est ainsi que naissent les écoles de commerce au Havre et à Rouen (1871), à Lille (1872), à Lyon (1872), Marseille (1872), ou encore à Bordeaux (1874), l'École des Hautes études commerciales de Paris (HEC en 1881). Les années 1900 voient l'apparition de plusieurs écoles de commerce réputées comme l'Ecole Supérieure de Commerce de Nantes (1900, devenue depuis Audencia Nantes), la HEC du Nord en 1906 (devenue en 1951 l'École des Hautes Études Commerciales du Nord, EDHEC) et l'Institut des Sciences Économiques et Commerciales (ESSEC, 1907).

Après la deuxième guerre mondiale, un décret ${ }^{61}$ précise un cadre institutionnel commun pour les écoles supérieures de commerce (ESC) reconnues par l'État : « les écoles supérieures de commerces reconnues par l'État sont des établissements d'enseignement technique supérieurs qui ont pour but de former les chefs des diverses entreprises commerciales ou financières et les cadres supérieurs de ces entreprises ou des services administratifs et commerciaux d'entreprises industrielles. » Toutefois, l'EDHEC (1906), l'ESSEC (1907), l'ISC Paris (1963), l'école des Hautes Etudes commerciales (HEC, 1881) ne sont pas soumises à ce cadre commun. Le statut et l'appellation des ESC tels que définis par cet accord disparaissent en $1991^{62}$, les écoles choisissent désormais librement leur nom et dispensent séparément leur diplôme.

Depuis plus d'une vingtaine d'années, les principales écoles de commerce font l'objet d'un classement annuel dans des magazines généralistes et spécialisés. Plusieurs écoles reviennent souvent en tête du classement, à savoir : l'Ecole des HEC (Paris), l'ESSEC, l'ESCP (Paris), l'EDHEC et Audencia (Nantes).

La renommée des écoles, la qualité supposée des enseignements dispensés ainsi que les profils des diplômés très proches des exigences des recruteurs expliquent en grande partie la facilité de l'insertion professionnelle des jeunes diplômés des grandes écoles y compris ceux des écoles de commerce malgré le contexte économique considéré difficile depuis quelques années. La dernière enquête annuelle sur l'insertion professionnelle réalisée par la Conférence des Grandes écoles (CGE) qui a porté sur les promotions de 2011 donne un taux d'emploi de presque $83 \%$ des diplômés des écoles de commerce (dont plus de $75 \%$ ont trouvé leur poste en moins d'un mois). Les résultats des diplômés managers sont moins bons que ceux des ingénieurs (85,7\% de taux d'emploi). L'insertion professionnelle dépend des domaines. En effet, les écoles de commerce forment à différents types de métiers comme la banque, la finance, les assurances, la gestion d'entreprise mais leur

${ }^{61}$ Décret n ${ }^{\circ} 47-2287$ du 3 décembre 1947.

${ }^{62}$ Décret n $91-785$ du 13 août 1991. 
diplômés se retrouvent également dans les secteurs du marketing et de la communication.

Il y a « de grandes disparités entre les quatre familles de métiers ouverts aux écoles de management. Le marketing et la communication sont particulièrement touchés par la crise. Il n'est pas rare que les diplômés mettent six mois voire un an pour y trouver un emploi. Une spécialisation pointue dans un domaine du marketing digital peut-être un atout supplémentaire, mais il faut savoir que la concurrence est très rude: la moindre annonce génère l'envoi de 200 à $300 \mathrm{CV}$. Les RH qui attirent un nombre croissant d'étudiants - peu inspirés par la vente ou la finance et découragés par le manque de débouchés dans le marketing - sont aussi en passe de se boucher. $»^{63}$

\subsection{Mutations de l'enseignement supérieur et professionnalisation}

Depuis plusieurs années, le système universitaire français montre plusieurs limites et pose plusieurs questions et débats. L'augmentation massive des étudiants dans l'enseignement supérieur n'est pas forcément synonyme de mixité sociale et de succès pour les classes sociales les plus défavorisées dont les enfants ont certes pu intégrer l'université mais n'y réussissent pas. En 2008, 35\% des élèves obtenant leur baccalauréat étaient enfants de cadres et de professions intellectuelles contre seulement 11,5\% qui étaient enfants d'ouvriers (Enquête MEN-MENSR-DEPP, 2009). La deuxième critique réside dans la dévalorisation des diplômes (Marie Duru-Bellat, 2006), par l'augmentation du nombre de diplômés notamment issus des formations généralistes. Mais l'élément de débat le plus important est le taux d'échec très élevé dans le premier cycle (environ $30 \%$ d'échec à la licence dans les formations généralistes), principalement parmi les étudiants issus d'un milieu modeste. A ce sujet, plusieurs travaux sociologiques (Place et Bruno, 2009; Tazouti, Flieller et Vrignaud, 2005) montrent qu'il y a, à l'évidence, des liens très étroits entre le parcours de formation, le niveau de diplôme et les habiletés scolaires du jeune, elles-mêmes en partie déterminées par l'origine sociale comme déjà prouvé par Bourdieu. Autrement dit, l'école non seulement ne gomme pas toutes les inégalités sociales mais elle peut les amplifier au travers du parcours de formation.

\footnotetext{
${ }^{63}$ Ginibrière Gaëlle, "Écoles de commerce : l'insertion professionnelle reste stable", Le Figaro, 14/11/12, disponible sur http://etudiant.lefigaro.fr/stages-emplois/trouver-un-job-stage-emploi/detail/article/ecolesde-commerce-1-insertion-professionnelle-reste-stable-452/ (consulté le 10/04/2013).
} 
Par ailleurs, l'enquête du CEREQ $^{64}$ qui permet de dégager des analyses comparatives entre les origines sociales des diplômés des grandes écoles et des diplômés d'IUT qui ont suivi par la suite un parcours universitaire $(\mathrm{Bac}+5)$ dans un cursus professionnalisant démontre que les origines sociales de ces jeunes jouent dans le choix du parcours universitaire. Si pour les diplômés de grande école de commerce le père occupe des fonctions d'artisan, de chef d'entreprise $(17 \%)$ ou de cadre (59\%), les diplômés issus d'un parcours professionnalisant viennent d'univers plus divers, leur père étant soit chef d'entreprise $(8 \%)$, cadre (53\%), employé $(15 \%)$ ou ouvrier $(8 \%)$. En rapport avec la théorie du déterminisme social, cette enquête soulève des questionnements quant à l'égalité des chances des jeunes dans l'accessibilité à certains diplômes réputés très reconnus sur la marché du travail mais qui demanderaient un investissement financier important, diplômes qui seraient réservés à certaines catégories sociales. Pour plusieurs spécialistes le problème réside dans l'orientation des étudiants et la sélectivité de certaines formations comme les IUT qui refouleraient les étudiants moyens au profit des meilleurs éléments. Les plus moyens se retrouvent donc confrontés au système universitaire classique auquel ils ne sont absolument pas préparés. D'un autre côté, l'université française s'est retrouvée confrontée aux difficultés de l'insertion professionnelle et de l'employabilité de ses diplômés en comparaison à ceux issus des grandes écoles. A ces raisons s'ajoutent l'augmentation de la capacité d'accueil des IUT, l'augmentation du nombre des grandes écoles et la création de plusieurs écoles de commerce et de management dont la sélectivité est moyenne pour expliquer la désaffection dont souffrent les cursus universitaires généralistes littéraires et scientifiques ainsi que la montée évidente de l'intérêt pour les formations professionnalisantes. Cette désaffection des cursus classiques et l'intérêt grandissant pour les formations professionnalisantes expliquent, entre autres, la réflexion dans le cadre du processus de Bologne ${ }^{65}$ et la mise en place depuis 2004 de la LMD dont le premier niveau de la licence est censé faciliter une insertion rapide des diplômés dans la vie active au bout de trois années de formation universitaire. En effet, "l'amélioration de l'employabilité des étudiants est centrale pour les universités qui doivent s'ajuster aux politiques de professionnalisation et pour les étudiants détenteurs de diplômes de valeur différenciée. » (Erlich et Verley, 2010, p. 79) Ainsi, les universités ont à leur tour renforcé leur offre de formations professionnalisantes telles que les licences professionnelles et les masters professionnels afin de consolider leur attractivité et de répondre aux exigences des recruteurs. Ainsi, plus de $40 \%$ des étudiants déclaraient en 2006 avoir fait un stage (en dehors du stage de fin d'études obligatoire dans plusieurs formations) contre moins d'un tiers en 1997.

${ }^{64}$ Centre d'Etudes et de Recherches sur les qualifications, enquête de 2008 sur les diplômés de 2005, 3 ans après la fin de leurs études.

${ }^{65}$ Processus ratifié en 1999 par les 46 pays européens participants. 
Avant de procéder à l'analyse des données permettant de comprendre l'insertion professionnelle des jeunes dans les métiers du marketing et de la communication, il nous paraît essentiel de situer notre propos dans un cadre théorique qui fait appel à des positionnements épistémologiques relevant de la sociologie du travail et de la compétence professionnelle. En sociologie du travail, nous pouvons faire appel à un cadre théorique mettant en exergue deux grandes théories, la théorie du capital humain et la théorie du signal et/ou du filtre. Puisque notre objectif est de traiter la performance de l'insertion professionnelle des jeunes issus des formations professionnalisantes revenir sur les théories de la professionnalisation nous paraît indispensable.

Du côté « du capital humain », selon les théories développées par Garcia-Aracil et Van der Velden (2008), on considère que le processus de formation participe à la construction de compétences. Des parcours différents (entre les grandes écoles et les formations universitaires professionnalisantes) pour le même niveau de diplôme impliquent des compétences différentes dues notamment aux concepts, aux méthodologies appliquées, ou encore aux pédagogies utilisées. Certaines de ces compétences considérées par les employeurs plus proches des performances exigées par le milieu professionnel seront plus valorisées en termes d'accès à l'emploi ou de rémunération. Dans ce sens, nous pensons que certaines formations universitaires professionnalisantes en dépit de leurs efforts d'intégration dans le tissu économique et de leur rôle fédérateur dans la professionnalisation du supérieur souffrent encore d'une mauvaise image chez les employeurs qui, dans les procédures de recrutement, préfèrent se tourner davantage vers les écoles supérieures privées.

Conformément à la théorie « du signal» (Eymard-Duvernay, Marchal 2000), les étapes du parcours de formation et le passage par des filières réputées sélectives, ainsi que l'obtention d'un diplôme connoté plus positivement (le diplôme de grande école) représentent des éléments d'information, des «signalements » émis lors du processus de recrutement et apportent aux recruteurs des informations discriminantes. La théorie économique du filtre, laisser penser que, dans les procédures de recrutement, les entreprises ont une préférence pour tout signal renvoyant à des formations sélectives qui sont réputées pour révéler des qualités ou des capacités qu'elles valorisent (efficacité, rapidité, réactivité). Les écoles de commerce, par les méthodes pédagogiques développées, les valeurs promues et une logique de professionnalisation de la formation rentreraient dans cette catégorie.

La problématique de la professionnalisation fait l'objet d'un vif débat non seulement dans le champ de la formation universitaire mais aussi dans celui du travail. Plusieurs sociologues du travail (Dubar, Tripier, 1989 ; Bourdoncle, 2000 ; Wittorski, 2007) ont abordé cette question mettant en exergue la réflexion universitaire autour de la professionnalisation de certaines formations, réflexion qui, selon ces auteurs, est à mettre en lien avec le discours des organisations sur la performance professionnelle et une certaine évolution du marché du travail qui nous 
oblige à repenser les rapports entre la formation universitaire et la compétence professionnelle. Ainsi, selon Wittorski (2007), le discours des organisations sur la professionnalisation est récent et il fait écho à plusieurs évolutions fortement articulées entre elles : le passage d'une logique de production poussée par l'offre (l'entreprise planifie le travail) à une logique de production tirée par la demande (les salariés sont invités à être « acteurs et auteurs du changement »), le passage à une logique du résultat, le passage d'un système contrôlé en son centre à une certaine décentralisation des responsabilités.

Cette évolution du rôle de l'employé dans l'organisation dictée notamment par des mutations profondes du marché du travail a été fortement prise en compte par les formations universitaires dans la réflexion menée autour de la professionnalisation de l'enseignement supérieur et de l'ancrage des formations universitaires dans le tissu économique français. Dans ce sens, le terme professionnalisation hormis les significations fort anciennes (professionnalisation de l'activité, professionnalisation du groupe exerçant l'activité-la création d'associations professionnelles) acquière de nouvelles acceptions indissociables de la réflexion universitaire menée à ce sujet. Ainsi, Bourdoncle (2000) dans ces travaux scientifiques sur la professionnalisation distingue trois autres objets de sens : la professionnalisation des savoirs (les savoirs professionnels ont tendance à être abstraits, organisés et validés selon un critère d'efficacité et de légitimité); la professionnalisation des personnes exerçant l'activité (c'est un processus d'acquisition de savoirs et de compétences professionnelles en situation réelle, le processus d'amélioration des savoirs et capacités et de construction d'une identité. Cela correspond à une dynamique de socialisation professionnelle); la professionnalisation de la formation (il s'agit de construire la formation de manière à ce qu'elle rende les individus capables d'exercer une activité économique déterminée). Cette nouvelle définition de la professionnalisation proposée par Bourdoncle vient appuyer la réflexion universitaire autour de la professionnalisation de l'enseignement et donnerait une valeur performative à l'insertion des jeunes diplômées sur le marché du travail. Autrement dit, l'insertion professionnelle passerait par une professionnalisation des savoirs transmis, plus proches et plus ancrés dans les logiques et le langage du milieu économique et par une acquisition des compétences et du savoir-faire en situation réelle. Dans ce sens tous les dispositifs de formation partenariaux et multiacteurs (stages, projets tutorés, missions en entreprises) jouent un rôle essentiel et représentent un critère de sélection majeur lors du recrutement. Selon le même auteur, la professionnalisation relève également d'une rhétorique et d'une dynamique de construction identitaire d'un groupe social. Cette stratégie appelée par Bourdoncle le "professionisme » c'est-à-dire la stratégie des militants à voir avec une dynamique de construction identitaire individuelle et collective. Au quotidien, la professionnalisation se construit ainsi par et dans l'élaboration identitaire qui dépend d'une reconnaissance par les autres des compétences et des savoirs produits. Cette 
définition s'appuie également sur le modèle de la construction identitaire de Dubar (1991) et se présente comme une dynamique fondée sur la projection dans l'avenir et sur la négociation dans un espace professionnel des modalités de reconnaissance et des objets reconnus (notamment les compétences). Cette conception consistant à associer étroitement professionnalisation et développement identitaire conduit à penser que les stratégies identitaires ainsi mises en œuvre participent de cette rhétorique soulignée précédemment et en sont même constitutives.

Les responsables des formations en communication suivent de près ces changements et s'adaptent aux impératifs de la professionnalisation et aux exigences de l'employabilité et des compétences exigées par les recruteurs. Plusieurs formations préparent aux différents métiers de la communication et il s'agit majoritairement de formations courtes.

\subsection{La formation aux métiers de la communication}

Plusieurs formations préparent aux métiers de la communication en fonction des niveaux de diplômes préparés. L'offre de formation universitaire propose plusieurs BTS de communication d'entreprise et de communication visuelle qui forment à un niveau $\mathrm{Bac}+2$. Les IUT proposent également des DUT en Information Communication qui sont reconnus pour être très sélectifs. Pour certains de ces DUT le recrutement se fait sur la base des dossiers et pour d'autres c'est une sélection sur dossiers et sur entretiens individuels (IUT du Havre) qui permettent de départager des candidats de bon niveau. Dans certains de ces départements d'IUT, la sélection est drastique étant donné le nombre limité de places. Au département Information Communication de l'IUT du Havre, l'option Publicité enregistre depuis 2008 une moyenne de 1200 candidatures pour 30 places et le département enregistre une moyenne de 2100 candidatures pour l'ensemble des trois options représentées avec un total de 90 places disponibles. Initialement créés pour accueillir les élèves issus de baccalauréat technologique, les départements Information Communication n'en prennent qu'un nombre très limité malgré les recommandations de réserver un tiers des places ouvertes aux détenteurs de baccalauréat technologique. Dans notre département, la proportion de Bac Technologique (principalement des bac STG) représentent moins du $1 / 5$ des candidats et ils représentent environ $1 / 5$ des effectifs recrutés (un quota de 7 étudiants sur une classe de 30). Par ailleurs, nos constatations à la rentrée universitaire nous indiquent que le nombre de candidats titulaires d'une mention Bien à Très bien dépasse le tiers des effectifs sélectionnés et c'est le cas de plusieurs autres départements Info Com.

Il y a aussi les licences professionnelles spécialisées et qui sont elles aussi très sélectives étant donné le nombre réduit de places (une moyenne de 25 places). 
Les universités offrent également des licences et des masters généralistes en Information Communication. On trouve aussi les écoles de communication publiques ou privées qui forment à un niveau $\mathrm{Bac}+3$ et $\mathrm{Bac}+5$.

A ces formations officiellement destinées à former les futurs professionnels de la communication, s'ajoute l'offre des écoles de commerce. D'où aujourd'hui une certaine concurrence entre les diplômés des formations professionnalisantes universitaires et ceux issus des écoles de commerce et dont la formation n'est pas forcément adaptée aux exigences des métiers et c'est ce que questionne notre contribution.

\section{Employabilité, insertion et mobilité : comparaison entre les formations professionnalisantes}

Cette étude comparative sur l'insertion professionnelle dans les métiers du marketing et de la communication concerne les diplômés des grandes écoles de commerce ainsi que les diplômés des formations universitaires professionnalisantes (IUT Bac+3). Afin de mener cette étude comparative nous nous basons sur les résultats de l'enquête réalisée en juin 2011 par l'ENSAI ${ }^{66}$ pour le compte de la Conférence des Grandes Ecoles sur les diplômés de 2010. Les résultats de cette première enquête (méthode de tris à plat et de tris croisés) seront croisés avec la synthèse des résultats de l'enquête réalisée par l'ADIUT en 2011 pour les diplômés de 2010 issus de formations professionnalisantes en marketing et communication d'IUT. Ces deux enquêtes poursuivent un double objectif : de mettre à disposition des institutions universitaires un outil d'aide au pilotage et de fournir une information nationale sur l'insertion professionnelle des jeunes diplômés dans les métiers du marketing et de la communication. Dans l'espace réservé à cet article c'est surtout ce dernier objectif qui fera l'objet de notre analyse comparative.

Parmi les nombreuses enquêtes réalisées sur l'insertion professionnelle des jeunes dans les métiers du marketing et de la communication, la similitude méthodologique (conditions de réalisation et items analogues) nous a conduites à choisir ces deux enquêtes. Avant de procéder à l'analyse comparative des deux enquêtes, quelques précisions d'ordre méthodologique s'imposent.

L'insertion professionnelle n'est pas mesurée après une période fixe suivant la fin de la formation, mais à partir de janvier quel que soit le mois de sortie. Dans les deux enquêtes, le traitement des données laisse une grande place à la comparaison : temporelle (20 années d'existence de l'enquête des grandes écoles et 11 ans pour les enquêtes d'IUT), genre (comparaison selon le sexe des diplômés), le type d'école (école supérieure de commerce, grande école de management, IUT).

${ }^{66}$ Ecole Nationale de Statistique et d'Analyse de l'Information. 
Concernant l'enquête réalisée pour les grandes écoles en 2011, nous précisons que parmi les 189 écoles concernées, membres de la Conférence des Grandes Écoles, 148 écoles ont accepté de participer à cette enquête (soit un taux de participation de $78 \%$ ). Sur les 31834 diplômés de ces écoles, 15771 diplômés ont répondu à l'enquête (soit un taux de participation de 49.5\%). Quant à l'enquête réalisée par l'ADIUT pour les diplômés de 2010, elle concerne 111 IUT dont 108 ont respecté le protocole imposé par l'ADIUT, la $\mathrm{DES}^{6768}$ et le CEREQ ${ }^{69}$ relatif aux contraintes de dates de retour, de récupération des questionnaires et de l'homogénéité quant à la qualité locale des retours. L'enquête concernait 45706 diplômés, dont 22799 ont répondu soit un taux de participation de $49.9 \%$.

Par ailleurs, afin d'obtenir des données d'analyse plus fines, non seulement sur le lien entre parcours universitaire et la performance de l'insertion professionnelle mais aussi sur l'évolution professionnelle de ces diplômés, nous nous appuyons également sur l'enquête réalisée sur un échantillon de 10000 diplômés par le CEREQ en 2008 sur les diplômés de 2005 trois ans après la fin de leurs études. Cette enquête permet de dire en quoi à diplôme égal $(\mathrm{Bac}+5$ pour les diplômés de grandes écoles et bac +5 pour les diplômés universitaires issus d'un parcours professionnalisant - DUT suivi de licence professionnelle et master professionnel en milieu universitaire) le parcours de formation constitue un facteur discriminant quant à la performance de l'insertion professionnelle. Cette étude menée sur plusieurs années permet également de questionner l'évolution professionnelle et de vérifier si le diplôme reste toujours un facteur discriminant entre ces deux catégories de diplômés. Cette tendance serait-elle inversée en termes d'évolution professionnelle où les réelles compétences et performance professionnelle contribuent à la création d'une identité professionnelle au-delà de l'effet discriminant du diplôme lui-même?

\subsection{Employabilité et insertion professionnelle}

Concernant la situation professionnelle des diplômés 10 mois après avoir fini leurs études, nous constatons un taux d'insertion professionnelle de $60 \%$ pour les diplômés des grandes écoles (niveau bachelor) contre 38\% pour les diplômés d'IUT. Alors que la poursuite d'études enregistre un taux de 50\% parmi les diplômés d'IUT, seulement $16 \%$ des diplômés de bachelor poursuivent leurs études. D'autres diplômés sont à la recherche d'un emploi : 19\% des diplômés de bachelor contre 6\%

\footnotetext{
${ }^{67}$ La participation à cette enquête réalisée par l'ENSAI et le taux de réponses des jeunes diplômés dépendent de l'implication et de la motivation des écoles et du groupe de travail auxquels participent régulièrement une cinquantaine d'entre elles.

${ }^{68}$ Direction de l'enseignement supérieur.

${ }^{69}$ Centre d'Etudes et de Recherche sur les Qualifications.
} 
des diplômés IUT. D'autres jeunes diplômés sont soit sans activité professionnelle ou sans projet professionnel clairement défini ( $6 \%$ des diplômés IUT contre seulement $2 \%$ des diplômés bachelor) ou font des missions de volontariat à l'international 3\% des diplômés bachelor.

Concernant la poursuite d'études après l'obtention d'un diplôme à niveau $\mathrm{BAC}+3$, parmi les diplômés d'IUT 4\% poursuivent une formation de niveau L1/ L2, $0.5 \%$ poursuivent une formation de type $\mathrm{DU}^{70}$ ou $\mathrm{DNST}^{71}, 10 \%$ des diplômés intègrent une licence de type L3, 7.5\% une licence professionnelle, 35\% un master professionnel, $12.5 \%$ une école d'ingénieurs, $5 \%$ une école de commerce et $16 \%$ poursuivent une autre formation. Quant aux diplômés des grandes écoles à niveau $\mathrm{BAC}+3$, nous constatons qu'ils privilégient essentiellement les études de type master dans le cadre des mêmes écoles. Ainsi, selon l'étude réalisée par l'ENSAI, $18 \%$ d'entre eux poursuivent un master spécialisé de la CGE, $0.7 \%$ un MSc de la CGE (master of science), $35 \%$ un master dans le cadre de leur école ou dans une institution similaire, 3.3\%, un master of science, 3\% un MBA (Master en Business Administration), 9\% passent des concours donnant accès à des écoles d'ingénieurs. Seulement $5 \%$ des diplômés de bachelor envisagent de faire une thèse et $25 \%$ des diplômés déclarent poursuivre d'autres types d'études. Contrairement aux résultats de l'enquête traitant de la poursuite d'études des diplômés d'IUT, l'étude réalisée par l'ENSAI fournit des éléments sur la poursuite d'études des diplômés des grandes écoles en fonction du paramètre genre. Nous constatons que, dans les choix de poursuite d'études, les hommes privilégient des masters spécialisés dans les écoles membres de la CGE, alors que les femmes sont un peu plus nombreuses dans les masters d'Etat et moins présentes dans les masters spécialisés de la CGE. A partir de l'analyse comparée des enquêtes réalisées en IUT et écoles de commerce, concernant la poursuite d'études nous pouvons dire que les jeunes diplômés poursuivent leurs études dans le même type de structures universitaires, les diplômés d'IUT privilégient les masters professionnels au sein des universités alors que les formations de type master en grande école attirent les étudiants diplômés de bachelor formés dans le même système universitaire.

Parmi les diplômés d'IUT à niveau Bac+ 3 (parmi ceux qui ont choisi de s'insérer sur le marché du travail sans poursuivre leurs études), nous constatons que l'insertion professionnelle est très rapide. 10 mois après avoir validé leurs diplômes, $89 \%$ des personnes interrogées ont déjà un emploi, $10 \%$ sont à la recherche d'un travail et $1 \%$ en inactivité. L'enquête réalisée par l'ADIUT révèle également que la durée de l'insertion professionnelle d'un diplômé ayant privilégié une licence

\footnotetext{
${ }^{70}$ Diplôme Universitaire.

${ }^{71}$ Diplôme National de Technologie Spécialisé.
} 
professionnelle est aussi longue que la durée de l'insertion professionnelle d'un détenteur de DUT.

Le cas des diplômés de niveau Bac +5 est relativement différent et cette tendance discriminatoire quant au parcours de formation se confirme à travers l'enquête menée par le CEREQ. Si les diplômés de grandes écoles réussissent à s'intégrer sur le marché du travail tout de suite après l'obtention de leur diplôme $(65 \%)$, seulement $53 \%$ des diplômés universitaires trouvent un travail sans passer par une période de chômage. 7\% des diplômés de grande école connaissent une période de chômage de 6 mois contre 16\% des diplômés issus d'un parcours universitaire. Le taux de chômage par comparaison entre ces 2 catégories de diplômés vient confirmer cette tendance discriminatoire à l'embauche à compétences et diplôme égaux. Si simplement 9\% des diplômés ESC sont au chômage un an après l'obtention de leur diplôme, $19 \%$ des diplômés universitaires sont à la recherche d'un travail un an après la fin de leurs études. Cet écart entre grande école et université au niveau de l'insertion professionnelle pourrait avoir deux explications : d'un côté le diplôme ESC est mieux côté sur le marché du travail parce que les dirigeants d'entreprise sont eux-mêmes issus de cette formation; d'un autre côté, les liens étroits entre ces écoles et le tissu économique notamment à l'occasion de divers projets mutiacteurs (que ce soit au niveau de l'enseignement et de la recherche) expliqueraient une préférence des recruteurs pour ces écoles.

Concernant la nature des contrats de travail proposés aux jeunes diplômés, une analyse croisée des deux enquêtes nous conduit à conclure que les diplômés de bachelor obtiennent plus facilement un contrat de travail à durée indéterminée, $78.1 \%$ contre $68.7 \%$ pour les diplômés de licence professionnelle. Cette insertion plus rapide sur le marché du travail s'expliquerait par le fonctionnement des grandes écoles basé notamment sur les partenariats avec les entreprises, l'insertion des professeurs permanents dans le tissu économique local ainsi que l'intervention de nombreux professionnels dans les formations de type master.

Néanmoins, l'enquête ENSAI comparant le taux de CDI de 2002 à 2010 montre une tendance à la réduction de la proportion des CDI à la faveur des emplois et statuts plus précaires. Cette tendance s'expliquerait par le contexte économique peu favorable qui entraînerait une plus grande exigence de la part des entreprises dans le recrutement en CDI. Par ailleurs, cette courbe descendante qui marque une précarisation des emplois lors de l'entrée dans la vie active, toucherait plus les femmes que les hommes. En effet, parmi les diplômés des écoles de management seulement $39.8 \%$ des femmes ont un emploi stables 10 mois après avoir validé leurs diplômes contre $60.2 \%$ des hommes. Les femmes sont deux fois plus nombreuses que les hommes en contrat à durée déterminée.

D'autres diplômés de grande école ou IUT entrent dans la vie active par des contrats précaires, des CDD, $15.8 \%$ des diplômés de bachelor contre $22.6 \%$ des diplômés de licence professionnelle. Les missions d'intérim enregistrent un très 
faible pourcentage pour les diplômés de bachelor qui ne représentent que $1.8 \%$ contre $5.6 \%$ pour les diplômés de licence professionnelle. 3.4\% des diplômés de bachelor ont des contrats type CDD ou CDI à l'étranger.

Cette différence se retrouve également au niveau du statut professionnel du diplômé. L'enquête du CEREQ nous éclaire à ce sujet. Ainsi, si $80 \%$ des diplômés ESC à niveau Bac +5 occupent un emploi de cadre, seulement $23 \%$ des diplômés universitaires au même niveau d'études ont le même statut professionnel. 18\% des diplômés ESC occupent des professions intermédiaires contre 39\% pour les diplômés issus d'une formation professionnalisante universitaire. D'après certaines études menées par le CEREQ (Calmand et Epiphane, 2010) l'origine sociale du jeune diplômé joue un rôle significatif dans l'accès à un emploi de cadre. Ainsi, de manière générale, il s'avère que, quel que soit le diplôme obtenu, les jeunes ont plus de chance d'accéder à un emploi de cadre si l'un des parents l'est. La même étude montre que si certains diplômes (diplôme d'ingénieur, IUFM) neutralisent l'effet de l'origine sociale du jeune diplômé, d'autres (et c'est notamment le cas des grandes écoles par comparaison avec le même diplôme obtenu dans un cursus universitaire professionnel) augmentent de manière significative ce rapport entre origine sociale et accès aux professions cadres.

Au niveau de la rémunération des jeunes diplômés, l'analyse des deux enquêtes révèle un écart au niveau des salaires entre les diplômés de grande école et les jeunes issus de licence professionnelle. Ainsi, si un diplômé de licence professionnelle touche en moyenne un salaire annuel net d'environ 18000/20000 euros selon les résultats de l'enquête fournis par l'ADIUT, un diplômé de bachelor dans le même domaine du marketing et de la communication démarrerait avec un salaire brut annuel se situant en moyenne à 31000 euros (sans primes). La même enquête révèle également une courbe descendante de la rémunération des diplômés des grandes écoles de 2008 à 2010 qui s'expliquerait par le contexte économique défavorable.

\subsection{Une lecture de genre}

L'élément genre, et en dépit de sa pertinence et de son caractère actuel, n'a pas été pris en compte dans l'enquête de l'ADIUT concernant la poursuite d'études des diplômés d'IUT ou leur évolution de carrière, alors qu'il apparaît comme un facteur d'explication dans l'étude réalisée par l'ENSAI et qui fournit des éléments sur la poursuite d'études, la nature des contrats et des postes des diplômés des grandes écoles. Nous constatons que, dans les choix de poursuite d'études, les hommes privilégient des masters spécialisés dans les écoles membres de la CGE, alors que les femmes sont un peu plus nombreuses dans les masters d'Etat et moins présentes dans les masters spécialisés de la CGE. Les femmes sont deux fois plus nombreuses que les hommes en contrat à durée déterminée. L'inégalité homme/femme se voit également au niveau de la rémunération. La courbe salariale étudiée par genre 
marque une disparité entre la rémunération des femmes et celle des hommes. Ainsi, si le salaire brut annuel (hors primes) d'un jeune diplômé de bachelor s'élève en moyenne à 33000 euros, le salaire moyen (hors primes) des femmes diplômées d'une école de commerce ne dépasse pas 30000 euros. Le peu d'éléments dont nous disposons actuellement dans l'analyse des tris croisés (niveau de rémunération/genre) ne nous permet pas de constater la même disparité au niveau de la rémunération des femmes issues de licences professionnelles. Ces constats rejoignent les inégalités salariales classiques en France tous domaines confondus. Les femmes sont généralement rémunérées $20 \%$ de moins que leurs collègues hommes à diplôme égal. L'écart est, toutefois, ici moins important que l'écart national puisque la différence est d'environ $10 \%$. Le marqueur genre est très important dans ce domaine où la majorité des diplômés est de sexe féminin. En DUT Information Communication les effectifs sont majoritairement féminins. A l'IUT du Havre (très semblable aux autres départements Info Com), nous avons connu des promotions où les classes de 30 ne comptaient qu'un ou deux étudiants hommes. Quand ils étaient nombreux, ils ne dépassaient pas les 6 étudiants sur une classe de 30. Une analyse plus approfondie sur la base d'enquêtes en IUT, qui prennent en compte ce paramètre genre et ses implications, peut ouvrir des pistes de réflexion et d'explication plus pertinentes.

\subsection{La concurrence dans les métiers de la communication}

Principalement formés aux métiers du marketing et du management, les diplômés des écoles de commerce et de management sont pourtant très nombreux à occuper des postes de cadres dans le secteur de la communication. Cette tendance se renforce depuis quelques années en dépit de la crise économique qui touche, entre autres, le secteur de la communication limitant ainsi relativement le nombre d'offres d'emploi. La forte présence des diplômés des écoles de commerce dans les métiers de la formation s'explique, pour plusieurs spécialistes et professionnels du secteur, par le flagrant manque de débouchés dans le marketing et la baisse de l'intérêt de ces diplômés pour la finance ou les métiers liés au système bancaire. C'est ainsi qu'ils seraient nombreux à s'orienter vers les ressources humaines et la communication même si ce dernier secteur souffre aussi de la crise économique. Par ailleurs, les postes disponibles aujourd'hui sont des postes avec une spécialisation élevée et pointue dans des domaines spécifiques comme le marketing mobile ou digital ou le e-commerce et la communication électronique sur les réseaux sociaux. Ces degrés de spécialisation sont possibles dans les Grandes écoles mais loin d'être offerts et assurés à un niveau DUT ou licence professionnelle, ce qui augmente la valeur et le caractère concurrentiel des diplômés des grandes écoles.

Cette situation questionne bien évidemment la problématique des compétences acquises et leur concordance avec les métiers exercés et les tâches à effectuer. Ceci 
nous amène à réfléchir autrement la question des compétences par domaine et le cloisonnement classique séparant le périmètre de compétences entre grandes écoles et formations universitaires classiques. L'organisation classique de la formation universitaire et la segmentation très marquée entre les différentes formations sont amenées à être revues, examinées et probablement corrigées étant donné l'évolution depuis quelques années du déroulement des carrières des diplômés issus des différentes formations (grandes écoles ou formations universitaires) et la mobilité de plus en plus présente entre les corps de métiers et vers l'étranger avec des conceptions et des pratiques professionnelles différentes et parfois très éloignées.

Voyant le nombre croissant des diplômés des grandes écoles dans les différents métiers et postes dans le secteur de la communication, plusieurs diplômés d'IUT et de licences professionnelles envisagent une poursuite d'études en écoles de commerce ou de management afin de décrocher un diplôme qu'ils considèrent comme une plus-value professionnelle. Notre charge de la direction des études du département Information Communication et l'aide apportée aux étudiants dans le choix entre les différentes poursuites des études possibles nous a permis de constater la mise en place, par nos étudiants, de nouvelles stratégies d'amélioration, stratégies qui sont fortement encouragées par plusieurs enseignants et professionnels intervenant dans la formation. Conscients de la concurrence existante avec les diplômés des grandes écoles et de la forte valeur des diplômes de ces derniers sur le marché du travail, certains diplômés des IUT s'orientent vers les écoles de commerce. Ils visent à les intégrer à niveau $\mathrm{Bac}+3$ afin de pouvoir prétendre à des postes occupés par les diplômés des écoles de commerce et de management et à des salaires qu'ils jugent «intéressants». Les 5 dernières années plusieurs de nos étudiants (y compris de l'option Métiers du livre) ont préparé et réussi, avec le soutien et l'aide des enseignants du département, les concours des écoles de commerce. Sur les 24 étudiants en $2^{\text {ème }}$ année de DUT Option Publicité, plus de 10 passent cette année plusieurs concours de ces écoles. Dans les entretiens que nous avons effectués, la concurrence avec les diplômés des écoles de commerce revient régulièrement. Nos étudiants enquêtés déclarent :

\footnotetext{
«Nous disposons des connaissances nécessaires mais on nous préfère les diplômés de commerce », «même si nous maîtrisons ce qui est demandé, les recruteurs optent pour des managers alors qu'il s'agit d'un poste de dircom", "je suis prêt à suivre des cours de gestion et de compta pour décrocher un diplôme qui a de la valeur sur le marché du travail ».
}

Les professionnels qui interviennent dans le cadre du DUT et les vacataires qui préparent les étudiants aux entretiens d'embauche et aux situations de recrutement ne leur cachent pas la difficulté du recrutement aujourd'hui face aux diplômés des écoles de commerce et les encouragent dans ce type de poursuite d'études en les orientant dans le choix des écoles de commerce et dans la préparation des concours. 
Nos étudiants sont également largement influencés par la réussite professionnelle des anciens diplômés de l'IUT qui ont obtenu des diplômes de commerce et de management. Lors de l'annuelle journée des anciens, organisée depuis plus de 5 ans par le département Information Communication de l'IUT du Havre, les étudiants comparent les parcours des anciens invités et ne peuvent occulter l'importance des postes et par conséquent des salaires des anciens du DUT désormais titulaires d'un diplôme en marketing ou management issus d'écoles de commerce comparé aux autres anciens ayant continué dans d'autres voies. La mobilité de ces anciens, leurs salaires et les courtes périodes de chômage qu'ils ont connues combinées aux recommandations et constatations des professionnels rencontrés lors des cours, confortent et encouragent nos étudiants dans le choix des écoles de commerce pour une poursuite d'études. Par ailleurs, nos étudiants sont plus nombreux à partir faire des stages à l'étranger ou y poursuivre leurs études et ceci en vue de renforcer leur $\mathrm{CV}$ et

« de lui donner une dimension internationale sur laquelle jouent beaucoup les diplômés de commerce », "si je ne fais pas mon semestre au Danemark, ce sera un IAE ou une autre école de commerce. Mes parents veulent s'assurer que je trouverai du travail quitte à payer une école pendant 3 ans. » Un étudiant affirme « J'ai acquis beaucoup de connaissances et de compétences dans le domaine de la publicité et du marketing, maintenant il faut les compléter avec un nom d'école vendeur et un stage à l'étranger ${ }^{72}$

Quand nous questionnons les 8 étudiantes en stage cette année au Canada, l'argument valorisation du CV face aux diplômés d'école de commerce revient chez 5 des 8 étudiantes. 6 d'entre-elles candidatent dans des écoles de commerce. La mobilité encouragée par l'IUT du Havre depuis plusieurs années, devient aujourd'hui une nécessité pour une majorité de nos étudiants. Ces derniers n'envisagent plus le DUT comme facilitant l'entrée rapide dans le marché du travail mais comme une première étape permettant l'acquisition des compétences techniques et pratiques indispensables dans la communication avant de décrocher «un diplôme plus à la mode et qui répond aux attentes des recruteurs eux-mêmes issus des écoles de commerce et de mangement $\gg$.

Les stratégies mises en place par nos étudiants (comme par d'autres) en termes de mobilité géographique à l'étranger pour les stages et les poursuites d'études rejoignent leur stratégie d'intégration d'école de commerce et de management dans l'objectif d'être aussi compétitifs que les diplômés de ces écoles en vue de décrocher des emplois dans le domaine de la communication et à des postes de responsabilités

\footnotetext{
${ }^{72}$ Entretiens réalisés entre octobre 2012 et mars 2013 avec des étudiants de l'IUT du Havre titulaires du DUT ou en $2^{\text {ème }}$ année de DUT.
} 
leur permettant une diversité des tâches et une certaine liberté de conception et de décision.

Les enseignants sont également amenés à adapter leurs méthodes pédagogiques pour se rapprocher du modèle de celles utilisées dans les écoles de commerce notamment par le recours grandissant aux études de cas. Les départements d'IUT ne peuvent toutefois faire appel à autant d'études de cas étant donné les coûts élevés d'abonnement aux centrales de cas. D'un autre côté, le département Information Communication du Havre, comme d'autres départements, a opté pour l'organisation de training et de challenge $e^{73}$ annuels afin de mieux préparer les étudiants aux situations de réponses aux commandes de professionnels dans des conditions très proches de la réalité des métiers. Ces exercices préparent les étudiants à la réalité de l'entreprise et surtout font connaître leurs compétences et leur formation auprès des professionnels qui sont des futurs recruteurs.

\section{Conclusion}

Malgré les limites méthodologiques du présent travail encore en phase exploratoire et la taille encore réduite de notre échantillon et des entretiens réalisés, nous pouvons affirmer certains résultats observés par ailleurs autrement ou sous d'autres angles dans d'autres études. Nous pouvons aussi affirmer que la concurrence entre diplômés des écoles de commerce et de management et les diplômés des formations professionnalisantes est une réalité qui amène les uns et les autres à adopter des stratégies de maintien (pour les étudiants en écoles) et d'amélioration (pour les titulaires de DUT) et des stratégies de contournement et d'amélioration pour les enseignants et les formateurs dans les formations professionnalisantes afin d'augmenter les chances d'insertion professionnelle de leurs étudiants et de renforcer leur compétitivité.

Cette évolution dans les conceptions mais aussi les évolutions enregistrées aujourd'hui dans le déroulement des carrières des diplômés toutes formations confondues (passage d'une spécialité à l'autre, perméabilité des secteurs d'emploi et spécialisation pointue pendant la carrière) nous amènent à engager la réflexion sur les notions de compétences et d'emplois et à envisager de les faire évoluer à la lumière de la réalité professionnelle : ce que nous envisageons dans la suite de cette contribution et avec l'apport de nouvelles enquêtes.

\footnotetext{
${ }^{73}$ Le training et le challenge sont des exercices en situation réelles auxquelles sont confrontés les étudiants du département Information Communication du Havre. Le training est un exercice de marketing et de communication hors médias réalisé en interne sur la base d'une étude de cas ou de la commande d'une entreprise locale. Le challenge est un exercice réalisé dans un contexte de compétition national ou régional. Les étudiants présentent leur réponse à la commande devant un jury composé par les commanditaires et des professionnels de la région.
} 


\section{Bibliographie}

Adangnikou N. (2007), Une évolution de l'efficacité de l'enseignement supérieur français : le cas des classes préparatoires scientifiques, Thèse de doctorat sous la direction de M. Duru-Bellat et J.-J. Paul, Université de Bourgogne, 339 p.

Béduwé C., Fourcade B., Giret J.-F., « De l'influence du parcours de formation sur l'insertion: le cas des diplômés scientifiques ", Revue française de sciences sociales, $\mathrm{n}^{\circ} 106$, avril-juin 2009, p.5-22.

Bourdoncle, Raymond, Autour des mots : professionnalisation, formes et dispositifs. Recherche et Formation, n³5, 2000, pp.117-132.

Calmand Julien, Epiphane Dominique, « Origine sociale et insertion professionnelle après des études supérieures : des diplômés plus égaux que d'autres », Net.Doc, $n^{\circ} 76,2010$.

Duru-Bellat Marie, (2006) L'inflation scolaire, Paris, Seuil.

Dubar Claude et Tripier Pierre (1998), Sociologie des professions, Paris, A. Colin.

Dubouis Hélène (2011), Les jeunes dans le monde du travail. Éd. Des Citoyens,

Erlich Valérie, Verley Élise. «Une relecture sociologique des parcours des étudiants français : entre segmentation et professionnalisation », Éducation et Sociétés, $\mathrm{n}^{\circ} 26,2010$, p.71-88.

Eymard-Duvernay F., Marchal E. (2000), « Qui calcule trop finit par déraisonner : les experts du marché du travail », Sociologie du Travail, vol. 42, pp. 411-432.

Garcia-Aracil A. Van der Velden R. (2008), Competencies for young european higher education graduates: labour market mismatches and their payoffs, Higher Education, vol. 55, n², pp. 219-239.

Ginibrière Gaëlle, «Écoles de commerce : l'insertion professionnelle reste stable », Le Figaro, 14/11/12, disponible sur http://etudiant.lefigaro.fr/stagesemplois/trouver-un-job-stage-emploi/detail/article/ecoles-de-commerce-1insertion-professionnelle-reste-stable-452/ (consulté le 10/04/2013).

Nicole-Drancourt Chantal (2006), L'insertion des jeunes en France, Paris, P.U.F., «Que sais-je?».

Place Dominique, Vincent Bruno (2009), «L'influence des caractéristiques sociodémographiques sur les diplômes et les compétences», Économie et statistique, $\mathrm{n}^{\circ} 424-425$, pp. 125-147.

Schwartz Barnard (2007), Rapport sur l'insertion professionnelle et sociale des jeunes. Apogée. 
Tazouti Youssef, Flieller André, Vrignaud Pierre (2005), «Comparaison des relations entre l'éducation parentale et les performances scolaires dans deux milieux socioculturels contrastés (populaire et non populaire) : Pratiques éducatives familiales et scolarisation $»$, Revue française de pédagogie, $\mathrm{n}^{\circ} 151$, p. 29-46.

Wittorski Richard (2007), Professionnalisation et développement professionnel, Paris, L'Harmattan.

Le livre blanc des IUT « Après 40 ans d'existence: Histoire, Bilan, Perspectives », Ed. 2007. 